\title{
A Missing Link in Galaxy Evolution: The Mysteries of Dissolving Star Clusters
}

\author{
Anne Pellerin ${ }^{1}$, Martin Meyer ${ }^{1}$, Jason Harris ${ }^{2}$, and Daniela Calzetti ${ }^{1}$ \\ ${ }^{1}$ Space Telescope Science Institute, 3700 San Martin Drive, Baltimore, MD 21218, USA \\ email: pellerin@stsci.edu, martinm@stsci.edu, calzetti@stsci.edu \\ ${ }^{2}$ Steward Observatory, 933 N. Cherry Ave., Tucson, AZ 85721, USA \\ email: jharris@as.arizona.edu
}

Keywords. galaxies: star clusters, stars: early-type, galaxies: evolution

Assuming that most stars form in clusters (Lada \& Lada 2003), one might initially expect to find most massive stars in stellar clusters and to detect most of the UV emission in young compact stellar clusters. However, Meurer et al. (1995) showed that in starburst galaxies only $20 \%$ of the UV emission at $2200 \AA$ is detected in clusters while $80 \%$ is diffuse emission from sources in the inter-cluster medium. This phenomenon is also observed for normal galaxies (Hoopes et al. 2001).

We performed PSF photometry on HST/ACS data of NGC1313 and constructed colormagnitude diagrams to identify the spectral types and luminosity classes of the most massive stars. Using this information, we created spatial maps for two groups of the most massive stars still on the main sequence: one with $\mathrm{F} 555 \mathrm{~W}<22.6 \mathrm{mag}$ and the second with $22.6<\mathrm{F} 555 \mathrm{~W}<$ 24.2 mag. Stars in the first group trace the morphological structures of the galaxy well and are mostly found in clusters. The cooler stars of the second group are significantly more scattered in the star field background. These stars are not found to be located in small clusters such as scaled-OB associations. Matching with evolutionary tracks, the second group of stars are early B-type stars, which live for 5 to 25 Myr. Since they were born in clusters, they must have left their birth place very rapidly. This is consistent with the infant mortality scenario (e.g. Fall et al. 2005). We think that these B-type stars found outside clusters are probably the stellar population dominated by B-type stars detected by Tremonti et al. (2001), Chandar et al. (2005), and Hoopes et al. (2001) in starbursts and normal spiral galaxies.

We also checked if the number of B-type stars in the star field background of NGC1313 is high enough to explain $80 \%$ of UV emission coming from outside the cluster environment. For each star on the CMD, we deduced its effective temperature and surface gravity, and then found its theoretical flux at $2200 \AA$ using stellar atmosphere models. We integrated the UV flux produced by the clusters and compared it to the UV flux produced by the stars in the field background. This calculation was done using various clusters definitions. For NGC 1313, we found $~ 90 \%$ of the stellar UV flux is produced outside clusters. We expect this fraction to be smaller for starbursts because of the larger number and size of clusters. Our results suggest that B-type stars are at the origin of the diffuse UV emission in galaxies, and that the infant mortality of star clusters is likely to be the cause of their homogenous distribution.

\section{References}

Chandar, R., Leitherer, C., Tremonti, C. A., Calzetti, D., Aloisi, A., Meurer, G. R., \& de Mello, D. 2005, ApJ 628, 210.

Fall, S. M., Chandar, R., \& Whitmore, B. C. 2005, ApJ 586, 939.

Hoopes, C. G., Walterbos, R. A. M., \& Bothun, G. D. 2001, ApJL 631, 133.

Lada, C. \& Lada. E. 2003, ARA $\& A$ 41, 57.

Meurer, G. R., Heckman, T. M., Leitherer, C., Kinney, A., Robert, C., \& Garnett, D. R. 1995, AJ 110, 2665.

Tremonti, C. A., Calzetti, D., Leitherer, C., \& Heckman, T. M. 2001, ApJ 555, 322. 